\title{
Editorial
}

\section{The Rise of Expanded Hemodialysis}

\author{
Claudio Ronco ${ }^{\mathrm{a}, \mathrm{b}}$

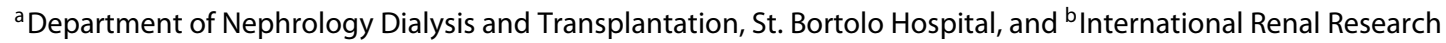 \\ Institute, Vicenza, Italy
}

\begin{abstract}
The low water permeability feature of original cellulosic membranes was considered an advantage in the absence of dialysis equipment that are capable of controlling water removal. The advent of ultrafiltration control systems led to the development and use of high-flux (HF) membranes that allowed improved middle molecule removal including $\beta-2$ microglobulin. Further advances in technology allowed better control over the structure and permeability of membranes. Different polymers and improved spinning modalities led to significant advances in solute removal and hemocompatibility. Inner surface modification produced a reduction in membrane thrombogenicity and protein-membrane interaction with a less tendency to fouling and permeability decay. Further evolution in technology led to the development of a new class of membranes referred to as protein-leaking membranes or super-flux or high cutoff (HCO). These membranes are more permeable than conventional HF membranes and allow some passage of proteins, including albumin. The rationale for these membranes is the need for increased clearance of low molecular weight proteins and protein-bound solutes. However, albumin loss in protein-leaking $\mathrm{HCO}$ membranes represents a limitation whose effect in patients is still controversial. The last evolution in the field of membranes is the development of a new class defined as "high retention onset" (HRO) due to the peculiar high sieving value in the middle to high molecular weight range. The introduction of $\mathrm{HRO}$ membranes in the clinical routine has enabled the development of a new concept ther-
\end{abstract}

apy called "expanded hemodialysis." Its simple set up and application offer the possibility to use it even in patients with suboptimal vascular access or even with an indwelling catheter. The system does not require particular hardware or unusual nursing skill. The quality of dialysis fluid is, however, mandatory to ensure a safe conduction of the dialysis session. This new therapy is likely to modify the outcome of endstage kidney disease patients, thanks to the enhanced removal of molecules traditionally retained by current dialysis techniques.

(c) 2017 S. Karger AG, Basel

\section{Introduction}

Significant improvements have been made to hemodialysis over the years leading to longer survival and improved quality of life in patients with end-stage kidney disease (ESKD). However, despite technological advances and improved patient care, long-term outcomes are still suboptimal with a high rate of hospitalization and mortality. This is partly due to a case mix, with older and sicker patients presenting a high prevalence of comorbidities. Nevertheless, important limitations of current dialysis techniques emerge clearly with several uremic solutes inadequately removed even by the most sophisticated renal replacement therapies [1-6]. While we are struggling with these challenges, health care systems are fixing boundaries to the overall expenditure and budgets, being con-

\section{KARGER}

(c) 2017 S. Karger AG, Basel

E-Mail karger@karger.com

www.karger.com/bpu
Claudio Ronco, MD

Department of Nephrology, Dialysis and Transplantation International Renal Research Institute of Vicenza (IRRIV)

San Bortolo Hospital, Viale Rodolfi 37, IT-36100 Vicenza (Italy)

E-Mail cronco@goldnet.it 
cerned about the growing costs of ESKD. Unexpected factors further complicate the above problems such as illegal immigration, inadequate investments and training for dedicated personnel, lack of resources, and incredibly high expectations from patients and their families.

\section{Challenges and Need for Innovation}

Several unmet clinical needs are still existent in current hemodialysis treatment methods. The evolution in technology represents a good opportunity to find solutions for unanswered questions. Thus, further innovation becomes mandatory. It can be incremental, slightly modifying the current technology with progressive improvement and refinement, or disruptive and revolutionary, leading to a quantum leap in technology and patient care.

Biomaterials and membranes with the contribution of new sciences such as microfluidics and nanotechnology represent areas where innovation is likely to occur in the coming years. We can anticipate further advances in patient care, thanks to treatment personalization and application of precision medicine. We might in fact improve our results by refining the analysis of the endotype and the genotype of specific patients and populations.

We think that investigators and scientists should use the experience of the past to imagine the future and shape the next generation of devices for renal replacement therapy. A careful analysis of the history of hemodialysis may help in this endeavor to avoid repeating the mistakes of the past and to project research and patient care toward more effective forms of treatments.

\section{Hemodialysis Therapy: A Never-Ending Journey}

Hundred years ago, Prof. Abel in Philadelphia performed an experimental mass separation between 2 fluid phases using celloidin tubes and called this process "dialysis." Thirty years later in Lund, Prof. Alwall performed the first blood cleansing session with his "artificial kidney." This represented a proof of concept, demonstrating the feasibility of hemodialysis. A few years later, Wilhelm Kolff created his rotating drum kidney that represented, both in the United Kingdom and in the United States, the first example of a reliable dialysis machine for chronic renal replacement therapy. Thus, hemodialysis was no longer experimental and the next challenge was to reach distributive justice providing renal replacement therapy to all patients who required it. The introduction of Scrib- ner's external shunt as chronic vascular access and the use of individual dialysis stations with incorporated singlepass dialysate preparation systems contributed to the expansion of the number of dialysis centers in the world and the number of patients on chronic hemodialysis. The issue of availability and access to therapy was then resolved, also thanks to the progressive reduction of dialysis treatment time from 12 to $4 \mathrm{~h}$ per session. This significant advance also made it possible to contribute to the full rehabilitation of many patients and their restitution to a life close to normal [7]. However, the reduction of treatment time required a remarkable increase in efficiency and a strict control of treatment tolerance. Blood flows up to $400-500 \mathrm{~mL} / \mathrm{min}$ and hemodialyzers with KoA close to 1,000 led to significant increase in treatment efficiency and urea clearances. In such conditions, the use of bicarbonate as a buffer became mandatory due to the slow conversion of acetate in the liver and consequent inadequate correction of acidosis. Dialysis machines were equipped with accurate pressure monitors, efficient blood pumps, well-dimensioned heaters, and more precise dialysate preparation systems with individualized concentrate solutions. Precise ultrafiltration control systems were required to modulate ultrafiltration in high surface area/ highly permeable hemodialyzers. This led to the discovery of significant side effects due to the backfiltration of contaminated dialysate into the blood and clearly revealed the requirement of high water quality and powder concentrates to avoid contaminations [8]. Several studies focused on dialysis quantification and adequacy in the presence of a reduced treatment time [9].

The National Cooperative Dialysis Study defined treatment adequacy only for small solutes and its mechanistic analysis [10] was based mainly on urea kinetics [11]. In spite of moderate toxicity, urea represents a suitable marker molecule, since its accumulation defines the need of dialysis, while its removal describes the efficiency of dialysis. The ratio between the product of clearance $x$ time and the volume of distribution of urea in the body $(\mathrm{Kt} / \mathrm{V})$ correlated with treatment outcome [10]. A $\mathrm{Kt} / \mathrm{V}<1$ was considered highly predictive of treatment failure. Based on these assumptions, several centers, especially in the United States, progressively reduced treatment time without considering, however, cardiovascular tolerance and other clinical aspects. Furthermore, double pool kinetics was not taken into account and consequently delivered Kt/V that was in most cases significantly lower than the prescribed value. The era of ultrashort dialysis treatments resulted often in catastrophic consequences. Fortunately, in the following years, better monitoring was
II

Blood Purif 2017;44:I-VIII DOI: $10.1159 / 000476012$
Ronco 
instituted and treatment time was adjusted for every patient considering double pool kinetics and tolerance to fluid withdrawal, leading to return in most cases, to $4 \mathrm{~h}$ per session. The effect of increased solute clearance, however, was absolutely out of discussion.

In spite of improved outcomes due to high efficiency hemodialysis, some complications of medium- and longterm hemodialysis remained unchanged. The theory behind this was a modern version of the Babb \& Scribner's square meter hour hypothesis [12] based on the accumulation of the so-called middle molecules. The concomitant observation that patients treated with adequate urea $\mathrm{Kt} / \mathrm{V}$ were displaying important complications such as anemia, skeleton and bone abnormalities, neuropathies, and carpal tunnel syndrome associated with dialysis-related amyloidosis (DRA), spurred new interest on analyzing molecules larger than urea and in particular beta-2 microglobulin ( $\beta-2 \mathrm{M})$. The improvement of DRA symptoms with the use of high-flux (HF) biocompatible membranes posed the question whether the positive results were dependent on the improved biocompatibility or the higher permeability of the new class of membranes (HF). We compared low-flux membranes with different biocompatibility levels (Cuprophan = low, Low flux polysulfone $=$ high) with HF membranes in regular dialysis and hemodiafiltration (HDF) to solve this problem. We concluded that convection (flux) was the determinant of $\beta-2$ $M$ reduction, while the contribution of biocompatibility and membrane composition was marginal [13]. In the Membrane Permeability Outcome study, we further corroborated these results showing the importance of convection and membrane permeability, especially in patients at high risk of complications (serum albumin levels lower than normal) [14]. These observations opened the pathway toward the use of HDF as a golden standard in chronic hemodialysis. Nonetheless, HDF is not approved in the United States; but it has provided important results in the United Kingdom and other countries where it is extensively applied. From the initial system of using a maximum of $9 \mathrm{~L}$ of reinfusion fluid provided in sterile bags, today's practice of online HDF (OL-HDF) has permitted to increase convective volumes up to 23-25 L per session [15]. This value has been correlated with significant improvement in survival and clinical outcomes [16]. Although online preparation of reinfusion fluid allows the possibility of carrying out simplified and optimized sessions, HDF still requires a series of technological steps to ensure the accuracy of fluid balance and the quality of replacement fluid made ultrapure by multiple online filtration steps.

The Rise of HDx
Specifically designed software, accurate monitoring devices, and efficient biofeedback techniques lead to the safe and effective conduction of the dialysis sessions and support the application of HDF in almost every center. However, considering OL-HDF as a major technological advance, clinical results and long-term outcomes of dialysis are still suboptimal and unsatisfactory. In particular, cardiovascular events are frequently encountered among the dialysis population and accelerated atherosclerosis that is associated with subclinical inflammation makes a significant impact on outcomes, thereby increasing hospitalization and affecting health care system budgets.

\section{The Unfolding Story of Uremia}

The complex story of uremia is slowly but progressively unfolding, thanks to the contribution of several investigators [17]. New retention molecules considered responsible for specific clinical outcome have been identified. In particular, high levels of PTH, FGF23, osteoclastin, osteoprotegerin, and other molecules involved in bone and calcium-phosphate metabolism have been associated with osteodystrophy. Hepcidin and proteins inhibiting bone marrow and erythropoiesis are considered responsible of uremic anemia. Homocystein and mediators of inflammation, including cytokines, have been identified as factors involved in accelerated atherosclerosis and cardiovascular complications. Leptin levels have been associated with a significant reduction of appetite. Recently, $\kappa$ free light chains $(22.000 \mathrm{Da})$ and $\lambda$ free light chains $(42.000 \mathrm{Da})$ have been identified as toxic molecules in the medium-high weight range [18]. Several of these molecules are well beyond the removal capacity of classic HF membranes and have molecular radii larger than that of membrane pores. We definitely need new options to take a step forward in enhancing the quality of blood purification techniques. This requires advanced research in the area of biomaterials and membranes but also the design of specific techniques to exploit the maximum benefits from new membranes and materials.

\section{Evolution of Dialysis Membranes}

When dialysis was first initiated, one of the most important parameters to define a membrane was the diffusive permeability $(\mathrm{Ko})$ as a function of thickness and surface area. Ko describes the capacity of small solute trans- 
Fig. 1. Theoretical sieving curves for 3 different classes of membranes: (1) high flux (HF), (2) high retention onset (HRO), and (3) high cutoff (HCO). The point in the curve where sieving coefficient is 0.1 determines the molecular weight cutoff (CO) value. The point in the curve where the sieving coefficient is 0.9 determines the molecular weight retention onset $(\mathrm{RO})$ value. It appears evident that HRO membrane, although presenting a similar cutoff value of the HF membrane, displays a completely different behavior. While the RO for the HF membrane is in the range of $1,200 \mathrm{Da}$ (Vit B12), the RO for the HRO membrane is in the range of $12,000 \mathrm{Da}(\beta-2$ microglobulin). MWCO value can be different in the 2 membranes with small leakage of albumin in the HRO class, but this effect is rapidly neutralized after a few minutes from the beginning of the treatment due to protein deposition at the blood membrane interface. HCO membrane has a $\mathrm{CO}$ value beyond the molecular weight of albumin and determines some albumin loss. Modified from Ronco and La Manna [33].

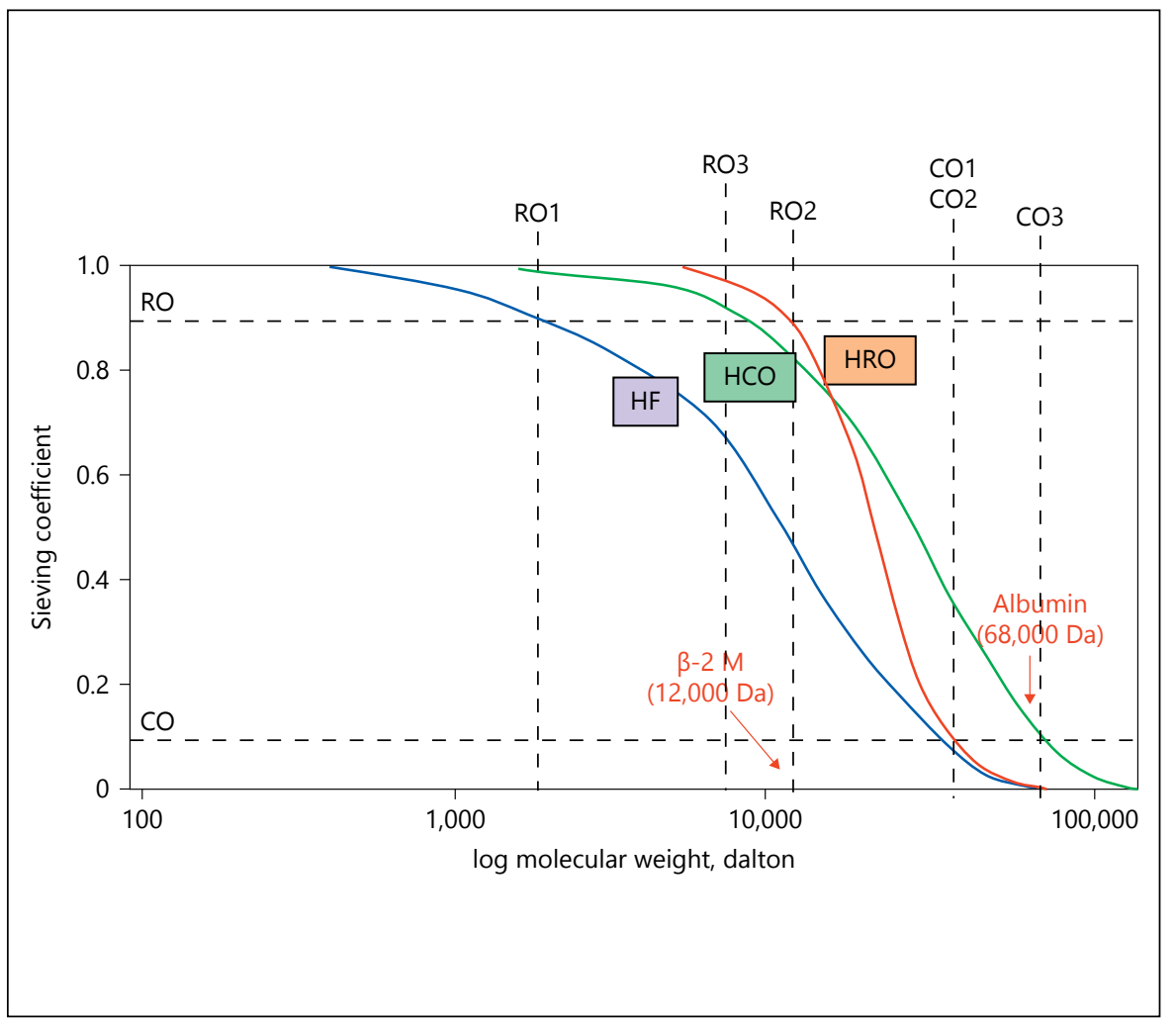

port in the presence of ideal conditions of diffusion gradient (theoretical conditions of unlimited blood and dialysate flow). The coefficient of diffusive permeability was extremely important in membranes with limited water permeability (low flux), displaying a significant correlation with the number of pores per unit of area, also defined as pore density. Subsequently, with the advent of HF membranes, new techniques such as hemofiltration and HDF were developed. In that case, where convective transport is remarkable, other parameters are utilized to classify a dialysis membrane [19].

Membrane performance may be characterized by hydraulic conductance (i.e., permeability to free water in the presence of a transmembrane pressure gradient), diffusion coefficient, and sieving properties (defined by the curve of sieving values observed for solutes in different molecular weight ranges). While the diffusion coefficient depends on pore density, the sieving curve mostly depends on the average pore size, that is, on the diameter of the channels crossing the internal skin layer of the membrane. The pore size distribution influences the cutoff value of the membrane (i.e., the molecular radius at which the sieving value results in the range of 0.1 ). This value is of quintessential importance when it comes close to the molecular radius of albumin, since the process of dialysis is essen- tially a size exclusion mass separation of solutes from blood and albumin loss should be carefully prevented.

Pore density and size distribution are the 2 main factors affecting the quantity and the spectrum of molecules that removed by a dialysis membrane. The sieving coefficient curve is an empirically derived function that depends on these variables. However, the cutoff value is insufficient to fully characterize the shape of the sieving curve. A further parameter termed "retention onset" (RO) should also be determined for this purpose. This parameter describes the molecular weight/radius where the sieving value is 0.9 . In an integrated transport model, a membrane with a tight pore size distribution will have a very steep sieving curve, while a membrane with a wide pore size distribution will have a flatter sieving curve possibly with a cutoff value beyond the molecular weight of albumin (Fig. 1). This new approach helps the characterization of new classes of membranes.

New membranes with a relatively high cutoff value have been recently introduced in clinical practice with the potential to remove toxins in the high molecular weight range that are increased in blood during sepsis, rhabdomyolysis, and hematological disorders. In such circumstances, high molecular weight solutes such as cytokines, myoglobin, or free light chains are the main targets for 
removal. The limit of these membranes, however, is the possibility of albumin leak due to the pore size opening and therefore their use is limited to a few sessions in diffusive mode until more information is available on clinical benefits and/or threats.

More recently, a great deal of effort has been made to produce membranes with a tight pore size distribution resulting in a steep sieving curve. The attempt is to keep molecular weight RO (MWRO) and molecular weight cutoff (MWCO) very close to each other, with a cutoff value close but lower than that of albumin. The result should be an improved removal of uremia retention molecules in the middle-to-high weight range (Table 1), with marginal or no albumin leak. Since this is an evolving area of research, we could imagine a new terminology to define and characterize this new class of membrane such as "extended sieving" or "expanded sieving" or something similar. However, based on these characteristics and the performance tests, we propose to define this new class of membranes as high RO (HRO) being the RO the new dimension of characterization (Fig. 2). The availability of this new class of membranes poses the question whether the technique terminology should also be updated. We can propose the new term "expanded hemodialysis (HDx)" for the application of HRO membranes in clinical dialysis. The term defines a treatment where diffusion and convection are conveniently combined inside a hollow fiber dialyzer equipped with an HRO membrane. A dialysis machine with ultrafiltration control is required, but no replacement solution or elevated ultrafiltration rates are needed to perform the therapy (Fig. 3).

\section{HDx: A New Therapy for a New Membrane}

The shape of the sieving curve of the HRO membrane is peculiar and optimized to perform HDx. Using a simple ultrafiltration-controlled hemodialysis technique, solute clearances in the spectrum of molecular weights traditionally retained with other techniques and membranes appear enhanced. Large molecules have low diffusion coefficients and their removal requires the contribution of convection. An increase in convection can be achieved either with HF membranes in HDF or by HRO membranes in HDx. In the first case, the clearance resulting from the product between ultrafiltration rate and sieving coefficient $(\mathrm{K}=\mathrm{Quf} \times \mathrm{S})$ is increased by increasing ultrafiltration (Quf) in the presence of a relatively low molecular sieving (S). In HDx, the same clearance is achieved in



Fig. 2. The tridimensional graph describes the domain map of hemodialysis membranes. Three main parameters describe the nature and performance of the membrane: (1) water permeability or flux (increasing this parameter we moved from low to high flux membranes), (2) membrane cutoff (MWCO), and (3) membrane retention onset (MWRO). The last 2 factors characterize the steepness of the sieving coefficient curve and its location in terms of molecular weight range. Modified from Ronco and La Manna [33].

Table 1. Ureamia retention solutes inadequately cleared by current hemodialysis techniques

\begin{tabular}{llll}
\hline Solute & MW, Da & Class & Action/effect \\
\hline$\beta-2$ M & 12,000 & & Amiloidosis CTS \\
Leptin & 16,000 & Middle* & Malnutrition \\
Myoglobin & 17,000 & & Organ damage \\
א-FLC & 23,000 & & Toxicity \\
Prolactin & 23,000 & & Infertility \\
Interleukin-6 & 25,000 & & Inflammation \\
Hepcidin & 27,000 & Large* & Anemia \\
Bound P-cresol & 33,500 & & CV toxicity \\
Pentraxin-3 & 43,000 & & Acute phase protein \\
$\lambda$-FLC & 45,000 & & CV toxicity \\
TNF- $\alpha$ (trim) & 51,000 & & Inflammation \\
\end{tabular}

* Value referred to the molecular weight interval between urea and albumin.

the presence of a much lower Quf because of higher S. In HDF, large amounts of ultrafiltration require replacement of volume by commercially prepared or fluids produced online. In $\mathrm{HDx}$, this is not needed. A significant amount of internal convection is present, but it is masked and balanced by an adequate amount of internal backfiltration. The mechanism of filtration-backfiltration is fur- 
Fig. 3. Expanded hemodialysis (HDx) and related operational parameters. Modified from Ronco and La Manna [33].



ther enhanced using fibers with reduced inner diameter leading to a high pressure drop in the blood compartment at a given blood flow. Thus, in the presence of enhanced sieving values for large molecules such as $\beta-2 \mathrm{M}$ or free light chains, relatively high clearances are achieved even at lower levels of convective flux and without requiring the fluid exchange volumes normally required in HDF. The fiber length and inner diameter are essential elements to optimize internal filtration and the mechanism of filtration-backfiltration. This mechanism, although invisible, makes it possible to achieve a significant amount of convection inside the dialyzer where filtration takes place in the proximal part and backfiltration compensates in the distal part. The ultrafiltration control system of the dialysis machine regulates the process and provides the exact amount of net filtration required for the scheduled weight loss of the patient [18-20]. There is no need for complicated equipment, but a blood flow of $300 \mathrm{~mL} / \mathrm{min}$ or higher and a dialysate flow of $500 \mathrm{~mL} / \mathrm{min}$ or higher are adequate. Due to the significant amount of backfiltration occurring in during treatment, water purity is an important requisite to avoid back transport of contaminants.

To cite a practical example we may use the graphs in Figure 1. Recent studies on HDF have suggested achieving at least $23 \mathrm{~L}$ of ultrafiltration per session [21-22]. Considering the molecular weight of $\beta-2 \mathrm{M}$ and the sieving properties of a standard HF membrane, total $\beta-2 \mathrm{M}$ clearance per session will be $23 \times 0.5=11.5 \mathrm{~L}$. To achieve the same result with an HRO membrane where $S=0.9$, you will only need $12.7 \mathrm{~L}$.

According to our previous studies [23-27], we could model the theoretical behavior of a $1.3-1.5 \mathrm{~m}^{2}$ dialyzer equipped with an $\mathrm{HRO}$ membrane in a reduced inner di- ameter configuration. A 1.5 dialyzer should be capable of generating an internal filtration of $40 \mathrm{~mL} / \mathrm{min}$ at zero net filtration. Assuming a dialysis session of $240 \mathrm{~min}, \mathrm{a} \mathrm{Qb}=$ $300 \mathrm{~mL} / \mathrm{min}$ and $\mathrm{Qd}=500 \mathrm{~mL} / \mathrm{min}$ and a scheduled fluid loss from the patient of $1 \mathrm{~L} / \mathrm{h}(16 \mathrm{~mL} / \mathrm{min})$, the internal filtration will be $56 \mathrm{~mL} / \mathrm{min}$ with a backfiltration of 40 $\mathrm{mL} / \mathrm{min}$.

Fifty-six mL/min will provide a total of $13,440 \mathrm{~mL}$ of filtration, which when multiplied by $0.9(\mathrm{~S})$ will produce an overall $\beta-2 \mathrm{M}$ clearance of $12.96 \mathrm{~L}$. The result is comparable and even superior to the numbers achievable with $\mathrm{HDF}$, with a simpler treatment and less technical requirements. This should be considered in addition to the possibility of achieving significant clearances of solutes such as $\lambda$ FLC whose clearance with HDF is marginal.

\section{Multidimensional Membrane Evaluation}

According to the above-mentioned approach, the evaluation of a membrane today should consider many different dimensions. In particular, as reported in Figure 4 , the dimensions could include the composition, the sieving $(\mathrm{S})$ for middle molecules such as B-2 M, the interaction with water molecules, the MWRO for different molecular weight solutes, the biocompatibility, the hydraulic conductance or permeability (Kf), the presence of electrical charges and potential, the MWCO for different molecular weight solutes, the thickness, and the diffusion coefficient (Ko). A specific graph should therefore be able to identify a membrane or a class of membranes and thus offer the needed information to clinicians for a correct application of the membrane in a defined technique and therapy. 


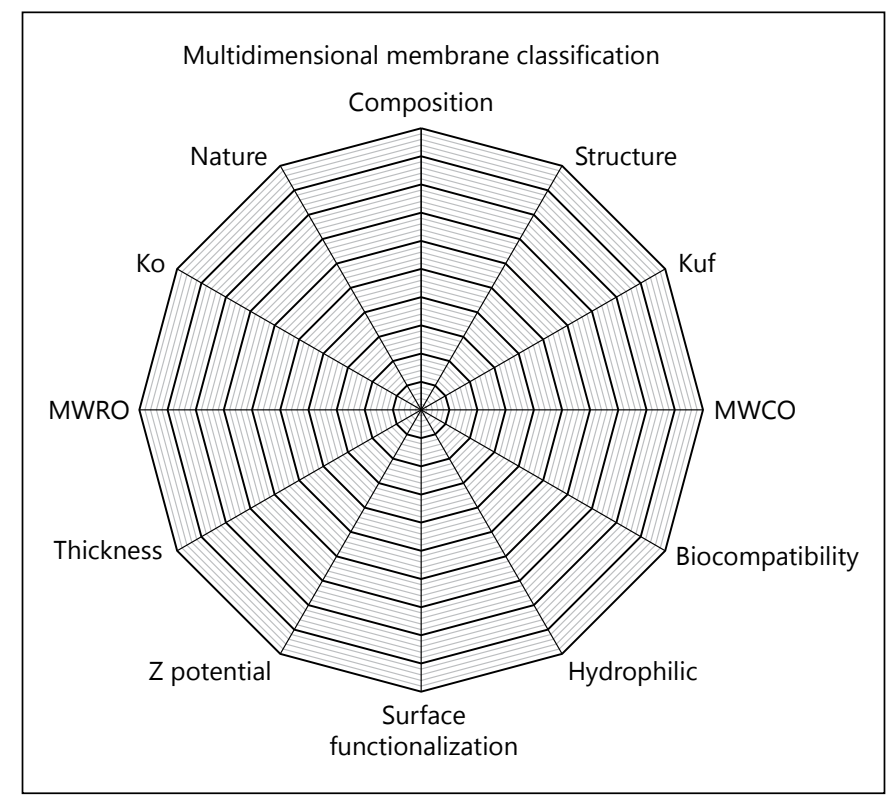

Fig. 4. Membrane class domain map. Multidimensional approach to membrane classification includes the composition, the sieving (S) for middle molecules such as B-2 M, the interaction with water molecules, the molecular weight retention onset (MWRO) for different molecular weight solutes, the biocompatibility, the hydraulic conductance or permeability (Kf), the presence of electrical charges and $\mathrm{Z}$ potential, the molecular weight cutoff (MWCO) for different molecular weight solutes, the thickness, and the diffusion coefficient (Ko).

\section{Conclusions}

The analysis of outcomes in clinical dialysis poses important challenges to the clinician and demonstrates that hemodialysis is still far from effectively replacing the function of the native organ. Nevertheless, innovation and research continue to propose new solutions and innovative devices even with caution for the occurrence of any possible side effects [28-32]. The case of HRO membranes and HDx is a typical example of progress and innovation in dialysis. It is unlikely that the efficacy of these membranes and this therapy will be proven in a large randomized controlled trial and we will probably base their application in clinical routine on criteria different from the classic evidence. Financial resources to perform a large and definitive trial involving thousands of patients are lacking. The low rate of events would require several years of observation of an everchanging case mix in which elderly patients are now present with high incidence and prevalence. Furthermore, the heterogeneity of the studied population and the different referral patterns will make it difficult to de- cide who should be the candidate for the trial and what the exclusion criteria should be. It will be probably easier to perform simple pragmatic trials utilizing registries and big data analysis derived from electronic medical records once HDx is sufficiently adopted in clinical routine. The selection of criteria for studies may result from cluster analyses or simply by the pathophysiological rationale for the application of the new therapy. One important question to define is the best utilization for HDx: is it going to be a rescue therapy for patients with a high level of uremia retention products, erythropoietin-resistant anemia, malnutrition-inflammation syndrome, and so on or is it going to be an elective therapy for patients beginning hemodialysis and candidates for an early transplant? HDx could be an ideal transition therapy for patients moving out from peritoneal dialysis and waiting for a transplant. This is an area where the application of precision medicine and treatment personalization will be highly recommended and will be found useful. The interesting features of HRO membranes and the improved possibility to remove middle-high molecular weight solutes will spur new research in the field of hemodialysis and will constitute a new hope for ESKD patients of improved medium- to long-term clinical outcomes. We will see in the next few years the "rise of HDx." It will all depend on the personal experience of the users as it happened in the past with innovations such as hemo-compatible HF membranes and bicarbonate dialysis. No randomized controlled trials have ever demonstrated their superiority compared to cuprophan or acetate dialysis, but the adoption of the innovation has been rapid and extensive. Nobody, except a few skeptical nihilists, would like to be dialyzed today with acetate dialysis and cuprophan membrane even in the absence of solid evidence.

\section{References}

1 Vanholder R, De Smet R, Glorieux G, Argilés $A$, Baurmeister $U$, Brunet $P$, Clark $W$, Cohen G, De Deyn PP, Deppisch R, Descamps-Latscha B, Henle T, Jorres A, Lemke HD, Massy ZA, Passlick-Deetjen J, Rodriguez M, Stegmayr B, Stenvinkel P, Tetta C, Wanner C, Zidek W: Review on uremic toxins: classification, concentration, and interindividual variability. Kidney Int 2003;63: 1934-1943

2 Neirynck N, Vanholder R, Schepers E, Eloot S, Pletinck A, Glorieux G: An update on uremic toxins. Int Urol Nephrol 2013;45:139-150.

3 Miyata T, Jadoul M, Kurokawa K, Van Ypersele de Strihou C: Beta-2 microglobulin in renal disease. J Am Soc Nephrol 1998;9:17231735. 
4 Cianciolo G, Colí L, La Manna G, Donati G, D'Addio F, Comai G, Ricci D, Dormi A, Wratten M, Feliciangeli G, Stefoni S: Is beta2microglobulin-related amyloidosis of hemodialysis patients a multifactorial disease? A new pathogenetic approach. Int J Artif Organs 2007;30:864-878.

5 Cianciolo G, La Manna G, Colì L, Donati G, D'Addio F, Persici E, Comai G, Wratten M, Dormi A, Mantovani V, Grossi G, Stefoni S: 5 -methyltetrahydrofolate administration is associated with prolonged survival and reduced inflammation in ESRD patients. Am J Nephrol 2008;28:941-948.

6 Desjardins L, Liabeuf S, Lenglet A, Lemke HD, Vanholder R, Choukroun G, Massy ZA; European Uremic Toxin (EUTox) Work Group: Association between free light chain levels, and disease progression and mortality in chronic kidney disease. Toxins (Basel) 2013;5:2058-2073.

7 Bonello M, Levin NW, Ronco C: History and evolution of the vascular access for hemodialysis. Contrib Nephrol 2004;142:1-13.

8 Ronco C, Brendolan A, Bragantini L, Chiaramonte S, Fabris A, Feriani M, Dell'Aquila R, Milan M, Scabardi M, Pinna V, La Greca G: Technical and clinical evaluation of different short, highly efficient dialysis techniques. Contr Nephrol 1988;61:46-68.

9 Collins A, Ilstrup K, Hanson G, Berkseth R, Keshaviah P: Rapid high-efficiency hemodialysis. Artif Organs 1986;10:185-188.

10 Gotch FA, Sargent JA: A mechanistic analysis of the National Cooperative Dialysis Study (NCDS). Kidney Int 1985;28:526-534.

11 Borah MF, Schoenfeld PY, Gotch FA, Sargent JA, Wolfsen M, Humphreys MH. Nitrogen balance during intermittent dialysis therapy of uremia. Kidney Int 1978;14:491-500.

12 Babb AL, Popovich RP, Christopher TG, Scribner BH: The genesis of the square meterhour hypothesis. Trans Am Soc Artif Intern Organs 1971;17:81-91.

13 Locatelli F, Mastrangelo F, Redaelli B, Ronco C, Marcelli D, La Greca G, Orlandini G: Effects of different membranes and dialysis technologies on patient treatment tolerance and nutritional parameters. The Italian Cooperative Dialysis Study Group. Kidney Int 1996;50:1293-1302.
14 LocatelliF, Gauly A, CzekalskiS, Hannedouche T, Jacobson SH, Loureiro A, Martin-Malo A, Papadimitriou M, Passlick-Deetjen J, Ronco C, Vanholder R, Wizemann V; Membrane Permeability Outcome (MPO) Study Group: The MPO Study: just a European HEMO Study or something very different? Blood $\mathrm{Pu}$ rif 2008;26:100-104.

15 Ronco C: Hemodiafiltration: evolution of a technique towards better dialysis care. Contrib Nephrol 2011;168:19-27.

16 Ronco C: Hemodiafiltration: technical and clinical issues. Blood Purif 2015;40(suppl 1): 2-11.

17 Barreto FC, Stinghen AE, de Oliveira RB, Franco AT, Moreno AN, Barreto DV, PecoitsFilho R, Drüeke TB, Massy ZA: The quest for a better understanding of chronic kidney disease complications: an update on uremic toxins. J Bras Nefrol 2014;36:221-235.

18 Zickler D, Schindler R, Willy K, Martus P, Pawlak M, Storr M, Hulko M, Boehler T, Glomb MA, Liehr K, Henning C, Templin M, Trojanowicz B, Ulrich C, Werner K, Fiedler R, Girndt M: Medium cut-off (MCO) membranes reduce inflammation in chronic dialysis patients-a randomized controlled clinical trial. PLoS One 2017;12:e0169024.

19 Ronco C, Crepaldi C, Brendolan A, Bragantini $\mathrm{L}$, d'Intini $\mathrm{V}$, Inguaggiato $\mathrm{P}$, Bonello $\mathrm{M}$, Krause B, Deppisch R, Goehl H, Scabardi A: Evolution of synthetic membranes for blood purification: the case of the Polyflux family. Nephrol Dial Transplant 2003;18(suppl 7): vii10-vii20; discussion vii55.

20 Fiore GB, Guadagni G, Lupi A, Ricci Z, Ronco C: A new semiempirical mathematical model for prediction of internal filtration in hollow fiber hemodialyzers. Blood Purif 2006;24: 555-568.

21 Maduell F, Moreso F, Pons M, Ramos R, Mora-Macià J, Carreras J, Soler J, Torres F, Campistol JM, Martinez-Castelao A; ESHOL Study Group. High-efficiency postdilution online hemodiafiltration reduces all-cause mortality in hemodialysis patients. J Am Soc Nephrol 2013;24:487-497.
22 Ronco C: Hemodiafiltration: evolution of a technique towards better dialysis care. Contrib Nephrol 2011;168:19-27.

23 Fiore GB, Ronco C: Principles and practice of internal hemodiafiltration. Contrib Nephrol 2007;158:177-184.

24 Ronco C: Fluid mechanics and crossfiltration in hollow-fiber hemodialyzers. Contrib Nephrol 2007;158:34-49.

25 Ronco C, Brendolan A, Lupi A, Metry G, Levin N W: Effects of a reduced inner diameter of hollow fibers in hemodialyzers. Kidney Int 2000;58:809-817.

26 Ronco C, Brendolan A, Feriani M, Milan M, Conz P, Lupi A, Berto P, Bettini M, La Greca G: A new scintigraphic method to characterize ultrafiltration in hollow fiber dialyzers. Kidney Int 1992;41:1383-1393.

27 Rangel AV, Kim JC, Kaushik M, Garzotto F, Neri M, Cruz DN, Ronco C: Backfiltration: past, present and future. Contrib Nephrol 2011;175:35-45.

28 Ward RA: Protein-leaking membranes for hemodialysis: a new class of membranes in search of an application? J Am Soc Nephrol 2005; 16:2421-2430.

29 Kirsch AH, Lyko R, Nilsson LG, Beck W, Amdahl M, Lechner P, Schneider A, Wanner C, Rosenkranz AR, Krieter DH: Performance of hemodialysis with novel medium cut-off dialyzers. Nephrol Dial Transplant 2017;32:165172.

30 Boschetti-de-Fierro A, Voigt M, Storr M, Krause B: Extended characterization of a new class of membranes for blood purification: the high cut-off membranes. Int J Artif Organs 2013;36:455-463.

31 Boschetti-de-Fierro A, Voigt M, Storr M, Krause B: MCO membranes: enhanced selectivity in high-flux class. Sci Rep 2015;5: 18448.

32 Foley RN, Parfrey PS, Harnett JD, Kent GM, Murray DC, Barre PE: Hypoalbuminemia, cardiac morbidity, and mortality in end-stage renal disease. J Am Soc Nephrol 1996;7:728736.

33 Ronco C, La Manna G: Expanded hemodialysis: a new therapy for a new class of membranes; in La Manna G, Ronco C (eds): Current Perspectives in Kidney Diseases. Basel, Contrib Nephrol, Karger, 2017, pp 124-133. 